\title{
El tiempo y el aprendizaje escolar la experiencia de la extensión de la jornada escolar en Chile*
}

\author{
SERGIO MARTINIC \\ Pontificia Universidad Católica de Chile, \\ Santiago de Chile, Chile
}

\section{RESUMEN}

En este artículo se ha analizado la importancia del tiempo como objeto de políticas de educación en América Latina y su impacto en el aprendizaje de los alumnos. Se ha presentado: la política de extensión de la jornada escolar (en el caso de Chile) y los cambios propuestos en la cantidad y la organización del tiempo en la escuela, en las prácticas de enseñanza, en las actividades estudiantiles y en las relaciones dentro y fuera de la escuela. Se han analizado los datos relacionados con la implementación de dicho programa, su eficacia e impacto en el aprendizaje. Se concluye que las principales lecciones aprendidas fueron la implementación de esta política y la necesidad de comprender la ampliación del tiempo en relación a los cambios en las prácticas de enseñanza de los docentes y en la organización cultural del tiempo en la escuela.

PALABRAS CLAVE

jornada escolar; interacciones profesor-alumno; aprendizajes.

* Este artículo se realizó en el marco del proyecto Tiempo escolar, intercambios y exigencia cognitiva en aulas de clase con alto y bajo SIMCE en segundo ciclo de educación básica. Proyecto n. 1110601 del Fondo Nacional de Desarrollo Científico y Tecnológico (FONDECYT). Sergio Martinic agradece también al Centro de Estudios de Políticas y Prácticas en Educación, Grant CIE01 de la Comisión Nacional de Investigación Científica y Tecnológica (CONICYT). 


\section{SCHOOL DAY DURATION AND LEARNING: THE EXPERIENCE OF THE EXTENSION OF THE SCHOOL DAY IN CHILE}

\section{ABSTRACT}

This paper analyzes the importance of time as an object of educational policy in Latin America and its impact in learning and in the integral development of students. It presents conceptual aspects of the relation between time and learning. The paper presents the implementation of the full-school-day program (JEC) in the educational c'hilean system. This policy supposes changes in the amount and management of time at school, in the pedagogical practices, in the student activities and in the internal and external school relationships. Data related to the implementation of JEC and its impact on learning is analyzed. The main lessons about the implementation of JEC and the necessity of understand it are closely related with changes in pedagogical practices of teachers and time management at schools.

KEYWORDS

full-school day; time management; teacher-pupils interactions; learning.

\section{TEMPO DE ESCOLA E APRENDIZAGEM: A EXPERIÊNCIA DA EXTENSÃO DO DIA NA ESCOLA NO CHILE}

\section{RESUMO}

Neste artigo foi analisada a importância do tempo como objeto de políticas de educação na América Latina e seu impacto na aprendizagem dos alunos. Foram apresentadas a política de estender o dia na escola, no caso do Chile, e as mudanças propostas na quantidade e organização do tempo de escola, em práticas de ensino, atividades estudantis e relacionamentos dentro e fora da escola. Analisaram-se os dados relacionados com a implementação desse programa, sua eficácia e impacto na aprendizagem. Concluiu-se que as principais lições aprendidas foram a implementação dessa política e a necessidade de compreender a extensão do tempo em relação às mudanças nas práticas de ensino dos professores e na organização cultural do tempo de escola.

PALAVRAS-CHAVE

tempo escolar; interações aluno-professor; aprendizagem. 


\section{INTRODUCCIÓN}

La organización actual del tiempo escolar responde a las características y realidad de la escuela del siglo XIX, propia de una sociedad estable, que tenía la tarea de transmitir conocimientos a una población restringida bajo el principio de que todo el mundo debía aprender lo mismo y al mismo ritmo.

La realidad social, la escuela y la noción misma de conocimiento han cambiado. Los sistemas educativos tienen amplias coberturas y atienden a una población de gran diversidad social y cultural. Las escuelas son más autónomas, interconectadas entre sí por redes informáticas y con una relación flexible a su entorno.

Pese a estos cambios, la organización del tiempo escolar sigue respondiendo a un concepto racional y mecánico tal como se definió cuando se organizaron las primeras escuelas en el siglo XIX. Los estudios demuestran que no existen en América Latina grandes cambios en la forma de organizar y gestionar el tiempo en las escuelas pese a las transformaciones de los sistemas educativos y del propio conocimiento a enseñar (Tenti, 2010). Como señala Husti (1992) el pensamiento sobre el tiempo escolar se ha detenido ante el cuadriculado horario de la planificación del tiempo, convertida en estereotipo y símbolo del trabajo escolar.

En los países de América Latina el promedio del tiempo escolar es cercano a 180 días y 800 horas al año. En Europa y Estados Unidos el promedio es de 840 horas al año y en el Sudeste Asiático es cercano a las 900 horas (Tenti, 2010).

Pero esta cantidad de tiempo disminuye al considerarse el tiempo real o efectivo de días y horas clases en un año escolar. En efecto, en muchos países los días efectivos no son más de 100 días al año y con frecuencia se pierde el 50\% o más de las horas asignadas para la enseñanza (Abadzi, 2007, 2009). Esto ocurre sobretodo en las escuelas públicas y de las áreas más apartadas. De este modo, los niños pobres de América Latina tienen poco tiempo de exposición a días y horas de enseñanza sistemática, lo que incide en los bajos resultados de sus aprendizajes.

Por otra parte, tanto profesores como estudiantes suelen demandar más tiempo para alcanzar los objetivos formativos propuestos. En América Latina las jornadas laborales de los profesores contemplan muy pocas horas de tiempo no lectivo. Ello afecta las horas dedicadas a la preparación de clases, trabajo individual, atención de alumnos y familias y reflexión pedagógica entre pares, entre otras actividades.

Si consideramos el tiempo desde el punto de vista de los estudiantes es evidente que no es suficiente para el estudio y el trabajo práctico en torno a materias; profundizar en aquellas que les son de mayor interés; reforzar las disciplinas difíciles de acuerdo al ritmo de aprendizaje de cada uno y para realizar actividades que vayan más allá de la escuela en función de sus intereses artísticos, deportivos y de desarrollo personal.

El problema se hace más complejo si consideramos que cualquier reforma o cambio educativo requiere de más tiempo. En efecto, las escuelas innovadoras necesitan de más tiempo para desarrollar sus proyectos de investigación, lectura individual, trabajo de equipo con otros profesores, atención diferenciada a los estudiantes, más interacción con los padres, etc. 
Hoy día se requiere de cambios profundos en la forma de organizar y gestionar el tiempo en las escuelas para responder a las exigencias de las transformaciones en el conocimiento; pedagogía e institucionalidad de los sistemas educativos.

La ampliación y mejor uso del tiempo escolar es uno de los cambios más importantes que afectan la cultura y gestión pedagógica de las escuelas. En los conceptos de tiempo y de espacio descansan los principios que separan las materias y regulan las relaciones entre los profesores y de estos con sus estudiantes. La ampliación de horas de clases; la disminución de otras; la incorporación de los tiempos que se requieren para llevar a cabo las innovaciones; las jerarquías internas; la disciplina; los espacios posibles para el trabajo en equipo y el cambio de prácticas en la sala de clases son cambios que afectan las jornadas de profesores.

\section{LA IMPORTANCIA DEL TIEMPO ESCOLAR}

La relación entre el tiempo escolar y el aprendizaje es una de las preocupaciones que ha tenido la investigación educacional. Los primeros estudios se basaron en el manejo del tiempo por parte del profesor y su efecto en la atención del estudiante. Esta perspectiva ha descansado en un concepto objetivo de tiempo definiéndola como una variable independiente que puede aumentar o disminuir físicamente, según las necesidades del sistema y del profesor. Una segunda tendencia considera el tiempo como un concepto subjetivo y relativo. En este caso el tiempo es una variable dependiente relacionada con las necesidades de los sujetos, las interacciones, contextos y culturas en las cuales estos se desenvuelven.

Detrás de estas perspectivas existen concepciones teóricas del tiempo las que, a su vez, descansan en supuestos epistemológicos más profundos que han alimentado la discusión en la ciencia social y que distinguen las tradiciones objetivistas de las subjetivistas (Friese, 1997). En palabras de Husti (1992) se trata de una discusión que distingue el tiempo como "cantidad" del tiempo como "significado".

Diversos estudios demuestran que las prácticas de los profesores siguen estando prisioneras del tiempo al desenvolverse bajo un sistema rígido de clasificación, secuencia y de orden. Los estudios demuestran que no existen grandes cambios en la forma de organizar y gestionar el tiempo en las escuelas, lo que afecta el logro de mejores aprendizajes.

La educación necesita un concepto de tiempo propio de nuestra época. Los tiempos disponibles y las clasificaciones actuales impiden realizar cambios en las prácticas pedagógicas que deben caracterizarse por la flexibilidad; considerar distintos ritmos de los estudiantes, culturas escolares, conocimientos, etc.

Desde nuestra perspectiva, contar con más tiempo asignado en la jornada escolar genera mejores aprendizajes siempre y cuando sea acompañado de tiempo efectivo dedicado a la tarea por parte de los estudiantes, de nuevas prácticas pedagógicas y de un reordenamiento de los medios didácticos, de las estructuras curriculares y de los contenidos educativos que se enseñan (Karampelas, 2005).

El tiempo escolar puede ser representado como una pirámide invertida. En la base superior se encuentran las unidades macro de tiempo (total de días y horas 
de clases en el año escolar), en el centro el tiempo asignado a actividades curriculares y en el vértice invertido de la pirámide el tiempo instruccional y comprometido para el aprendizaje en la sala de clases (Aronson; Zimmerman; Carlos, 1998).

Desde esta perspectiva no basta con aumentar la oferta de horas de enseñanza a nivel macro para mejorar los aprendizajes. Es necesario garantizar que los estudiantes tengan un compromiso con la tarea y ello implica entender sus ritmos, conocimientos previos, motivaciones y, sobre todo, poner en práctica una metodología atractiva y activa en la sala de clases (Belleï, 2009; Bloom, 1974; Delhaxhe, 1997; Karampelas, 2005; Wubbels; Brekelmans, 2005).

\section{EFECTOS EN LOS APRENDIZAJES}

La investigación ha demostrado que el tiempo es una variable que afecta positivamente el rendimiento y aprendizaje de los estudiantes. De ahí que muchos países han optado por extender las jornadas diarias de los estudiantes en el establecimiento y/o el largo del año escolar, bajo el supuesto que más tiempo del alumno en la escuela generará mejores logros académicos.

En general, la evidencia muestra una asociación positiva entre tiempo y logro académico, pero advierte que la magnitud del efecto depende en gran medida del uso que se haga de ese tiempo y de la interacción que se establezca entre el/la profesor/a y el/la alumno/a. Existe una relación consistente entre la cantidad de tiempo asignado a la instrucción y la cantidad de tiempo que los estudiantes ocupan participando efectivamente en actividades de aprendizaje (Aronson; Zimmerman; Carlos, 1998). Por lo tanto, cuando el tiempo es bien usado (es decir, una gran proporción está destinada a la enseñanza y la instrucción), el tiempo asignado tiene efectos positivos en el aprendizaje. Por el contrario, cuando la mayor parte del tiempo se usa en situaciones distintas a la instrucción y la enseñanza, la extensión de la jornada o del tiempo asignado no producirá ganancia alguna (idem).

La investigación internacional muestra que alumnos que asisten a establecimientos que han aumentado su jornada diaria escolar tienden a obtener mejores logros académicos, medidos generalmente con pruebas estandarizadas de aprendizaje (Redd et al.,2012). No obstante, estos estudios no logran concluir sobre el efecto directo de la extensión de la jornada en el logro académico, ya que muchos de esos programas son parte de intervenciones mayores, por lo que es muy difícil aislar los efectos.

Los estudios concuerdan en que los estudiantes provenientes de familias de nivel socioeconómico bajo se benefician más de la extensión de la jornada (idem). Una investigación realizada en Uruguay concluyó que aquellos/as alumnos/as provenientes de familias de nivel socioeconómico bajo que asisten al programa de jornada escolar completa ( 8 horas diarias) tienen un $10 \%$ más de probabilidad de pasar de curso en tercero básico que sus pares que asisten a jornada escolar regular (3 a 4 horas diarias) (Cerdan-Infantes; Vermeersch, 2007). Llama la atención que el programa de jornada escolar completa en Uruguay ofrece a los/las alumnos/as actividades extra curriculares más que un aumento de actividades instruccionales. El efecto que se observa es modesto y los autores sugieren que la extensión de la 
jornada tendrá efectos limitados sin un aumento en el efectivo compromiso de los/ las alumnos/as en las tareas de aprendizaje.

La evidencia también muestra que los efectos del aumento de asignación de tiempo en el aprendizaje son mayores en países menos desarrollados. Un experimento natural conducido en Etiopía analizó el efecto de la jornada diaria completa sobre el aprendizaje en niños y niñas de primero y segundo básico, usando como grupo control establecimientos que permanecen con jornada parcial. Los resultados muestran un efecto positivo significativo en el aprendizaje de las matemáticas (Orkin,2011). En Argentina, un estudio longitudinal sobre los efectos a largo plazo de la extensión de la jornada escolar implementada en 1971 no observó impacto directo en el aprendizaje, pero sí comprobó que los/las alumnos/as que asistieron a jornada completa en educación básica obtuvieron una tasa de graduación en la educación secundaria $21 \%$ más alta que aquellos que permanecieron con jornada parcial. El autor señala que este resultado es explicado principalmente por el efecto observado en los alumnos provenientes de familias de nivel socioeconómico bajo (Rodriguez, 2009).

La evidencia sobre los efectos de la extensión del año escolar es más controversial. Experimentos naturales y cuasi-experimentales también han comprobado una asociación positiva entre ambas variables, pero no tan sistemática como el efecto de la extensión de la jornada diaria.

En una revisión sistemática, Redd et al. (2012) encontrado que 18 de 28 estudios demostraron un efecto positivo de la extensión del año escolar en logro académico y porcentaje de asistencia de los alumnos. Hay otros estudios que no encuentran asociación alguna y dos estudios muestran efectos negativos. Un estudio conducido sobre una muestra representativa de cursos de segundo medio en Estados Unidos concluyó que el aumento del año académico tiende a incrementar el rendimiento escolar en alumnos con bajos resultados previos, pero provoca el efecto contrario en alumnos que presentan mejores resultados en pruebas de aprendizaje estandarizadas (Eren; Millimet, 2007). Por otra parte, un estudio del Banco Mundial realizado en India señala que las escuelas con mejor rendimiento escolar ofrecen una jornada de 66 horas más al año que las escuelas con menor rendimiento escolar (Banco Mundial, 1997 apud Abadzi, 2009).

\section{EL TIEMPO COMO OBJETO DE POLÍTICAS}

Hasta comienzos de la década de 1980 el problema de la organización del tiempo y de la jornada escolar no era una preocupación relevante para los tomadores de decisiones en América Latina.

En las reformas de la década de 1990, en cambio, el tema adquiere importancia ya que los sistemas educativos se plantean metas más ambiciosas de enseñanza y de aprendizajes y que requieren de más tiempo para obtener buenos resultados.

El informe "Prisioneros del tiempo" (Prisoners of time), realizado por la National Education Commission on Time and Learning (1994), tuvo un gran impacto en los Estados Unidos y llamó la atención sobre el problema a nivel 
internacional. Desde entonces, tanto en Estados Unidos como en Europa comienzan a desarrollarse políticas orientadas a mejorar la eficiencia en el uso de tiempo y a ampliar la jornada escolar.

En los países de la Organización para la Cooperación y el Desarrollo Económicos (OCDE) existe una tendencia a no extender la jornada escolar más allá de las cinco horas diarias en los primeros años de la escuela primaria y no mayor a seis horas en la escuela media inferior y superior. Se asume que, para mejorar el aprendizaje no basta solo más horas sino que, además, es necesario un aprovechamiento óptimo del tiempo escolar a través de un buen trabajo pedagógico.

En América Latina la situación es muy heterogénea y los tiempos disponibles y reales varían de acuerdo a los países, el nivel socioeconómico de la población y el carácter rural o urbano de las escuelas.

Los estudios realizados confirman diferencias entre el tiempo oficial o asignado y los días efectivos de clases. En América Latina, pese a los 180 días de clase promedio y oficiales, en muchos países no se realizan más de 100 días y se pierde el $50 \%$ o más de las horas asignadas para la enseñanza. Los conflictos y huelgas prolongadas de profesores y estudiantes, las licencias médicas, desastres naturales, entre otros, son factores que inciden en la disminución del tiempo efectivo de clases en América Latina. Esta situación afecta principalmente a los niños y jóvenes de los quintiles más pobres de la población.

En varios países de la región se han iniciado políticas de extensión de la jornada escolar orientadas, fundamentalmente, hacia los sectores más desfavorecidos. Con ello se pretende ampliar las oportunidades educativas que permitan la adquisición de saberes relevantes y complejos. A través de estas políticas, a su vez, se espera alcanzar otros objetivos asociados a la socialización de los niños, garantizar su alimentación y generar oportunidades para su desarrollo integral a través del arte y el deporte.

Entre estas experiencias cabe mencionar Argentina, Brasil, Colombia, Chile, México, Uruguay y Venezuela, entre otras. Con diferentes estrategias y coberturas estas políticas se han planteado intervenir sobre el tiempo escolar y su propia organización para mejorar los procesos pedagógicos y garantizar aprendizajes equitativos. Con ello se pretende que las escuelas cuenten con más tiempo para aplicar un currículo más complejo y extenso y para que los profesores y alumnos tengan tiempo para su desarrollo, trabajo individual y actividades con sus pares (Tenti, 2010; Vercellino, 2012).

La escuela al disponer de mayor tiempo puede desarrollar mejor su función social, distribuir sus actividades escolares, profundizar más los conceptos, abordando diferentes estrategias e ir incorporando la realidad al análisis académico.

Estos cambios son difíciles y suelen ser muy conflictivos en el ámbito educativo. Todo cambio en el horario escolar implica un cambio en el ritmo de vida y coordinaciones de muchas personas, tanto en el alumnado como de las familias y el profesorado; cambio en el funcionamiento de la economía y de la vida cotidiana de todos quienes interactúan con la escuela. 


\section{LA EXPERIENCIA EN EL CASO DE CHILE: JORNADA ESCOLAR COMPLETA}

En Chile se aprobó en el año 1997 la Ley de Jornada Escolar Completa (JEC), que aumentó en un 30\% las horas de clases en la enseñanza básica y media del país. Con esta política, los establecimientos subvencionados públicos y privados alcanzan un total de 1.100 horas anuales cronológicas de clases desde tercer a octavo básico superando la media de los países de la OECD. La incorporación ha sido gradual y en el año 2012 cerca del $90 \%$ de los establecimientos subvencionados se encuentran adscritos a este régimen de jornada escolar (Brunner; Elacqua, 2006; Cox, 2005; Valenzuela, 2005).

Desde 1997 se han incorporado gradualmente las escuelas y liceos a este régimen de jornada. En la casi totalidad de los casos la implementación de este régimen ha contado con la construcción y/o ampliación de la infraestructura de los establecimientos. En la actualidad cerca del $90 \%$ de los establecimientos del país tienen este régimen.

Toda política descansa en una teoría de cambio que relaciona o encadena diferentes factores en función de logros intermedios y finales medidos usualmente a través de los aprendizajes de los estudiantes. Estas son las hipótesis de cambio que tienen sustento en el conocimiento acumulado y en la experiencia práctica de la investigación y múltiples programas similares. Se construye así un cuerpo de ideas que fundamenta y da racionalidad a la política o iniciativa que se promueve (Mokate, 2000).

El objetivo fundamental de la JEC -tal como se enuncia en sus fundamentoses la reestructuración del uso del tiempo en las escuelas para ponerlo al servicio del mejoramiento de los aprendizajes y de la formación de los alumnos y de las alumnas.

La Figura 1 representa las relaciones básicas que dan cuenta de las relaciones y su encadenamiento para producir los cambios esperados y permite visualizar la interacción de los distintos elementos o componentes de la estrategia del programa ordenados en función de tres grandes categorías: insumos, procesos y resultados.

Los insumos aluden tanto al contexto en el cual se desarrolla el programa como a los recursos de entrada que aporta el sistema educativo. Los procesos dan cuenta del uso y efectos que tienen tales insumos en las prácticas e interacciones de profesores entre sí y con sus alumnos. Por último, los resultados aluden a los logros obtenidos y que se expresan en aprendizajes cognitivos y sociales, cambios en el desarrollo profesional de los docentes y en el clima y cultura de la escuela.

La hipótesis central de programa es que más tiempo en la escuela significa mayor posibilidad de aprender. En efecto, se sostiene en los fundamentos que si aumentan los tiempos pedagógicos mejorarán los aprendizajes favoreciendo a las escuelas más postergadas que, además de contar con más tiempo, mejoran su infraestructura y planta docente (MINEDUC, 2002). Gran parte de la justificación del diseño de la JEC descansa en esta hipótesis. Si los alumnos tienen más tiempo de exposición al aprendizaje ello tendrá efectos en sus resultados. 


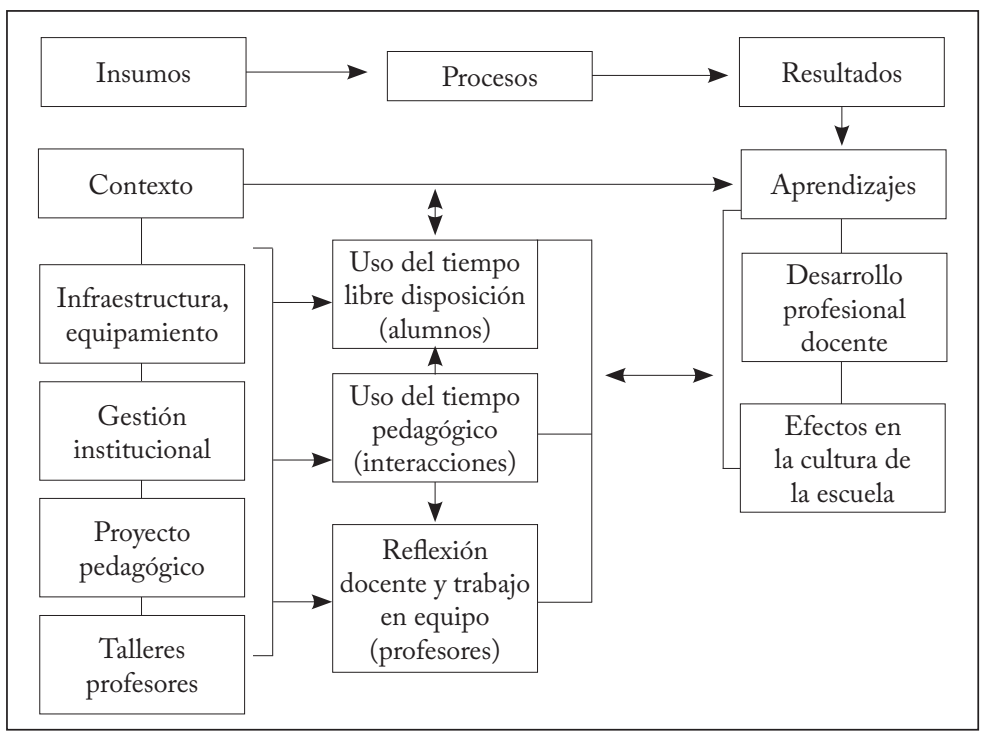

Figura 1 - Modelo de relaciones Programa Jornada Escolar Completa.

Fuente: Martinic; Huepe; Madrid (2008, p. 126).

Elaboración propia.

Para alcanzar los resultados esperados se requieren de insumos y de procesos. Los primeros dan cuenta de las condiciones y de los recursos humanos y materiales que aporta la política y que deben existir en los establecimientos. Los segundos dan cuenta de los procesos internos, y fundamentalmente pedagógicos, que se espera desarrollar para lograr un mejoramiento de las prácticas pedagógicas y obtener mejores resultados de aprendizaje.

\section{LA IMPLEMENTACIÓN DE LA POLÍTICA}

La integración de los establecimientos a este régimen de jornada de trabajo ha sido gradual principalmente por problemas en la infraestructura. La JEC ha requerido de una intervención e inversión en infraestructura, especialmente en aquellas unidades educativas que funcionaban en doble jornada y que no poseían la capacidad física de acoger a la totalidad de sus alumnos en una jornada única. Esta intervención supone velar por la adecuación de las salas de clases, la generación de espacios de circulación, recreación y servicios de apoyo.

El Estado ha apoyado a los establecimientos públicos y privados con inversiones en infraestructura asociadas a la extensión de la jornada. Para esto cuenta con un sistema de apoyo a la inversión que consta de dos componentes fundamentales: preinversión ${ }^{1}$ y costo de capital asociado a la nueva infraestructura (aporte suplementario

1 Asistencia técnica en la preparación de proyectos de inversión para los establecimientos educacionales que requieran expandir su infraestructura la que se concentra en las etapas de identificación y elaboración de los proyectos de inversión. 
por costo de capital adicional). Gran parte de los recursos de la política se han concentrado en la infraestructura y equipamiento necesario para la implementación de la JEC. Este ha sido uno de los resultados más exitosos y la opinión pública tiende a relacionar JEC con construcción de establecimientos más que con reordenamiento del uso del tiempo escolar. Existe una percepción muy positiva del diseño y de la calidad de la infraestructura de los establecimientos incorporados a la JEC lo que cambió el paisaje de las escuelas públicas urbanas y rurales a lo largo del país.

En relación a la gestión institucional, los establecimientos educacionales en JEC extendieron el tiempo de acuerdo a las normas definidas en la ley (ley n. 19.532, de 1997). Estas normas se acogen y formalizan en los proyectos pedagógicos de JEC que cada establecimiento educacional se compromete a ejecutar al ingresar a este régimen.

Estos proyectos revisan y rediseñan la organización de las actividades de enseñanza y aprendizaje. Identifican problemas y proponen estrategias y prioridades para enfrentarlos. El proyecto debe ser consistente con el Proyecto Educativo Institucional, y con los requerimientos pedagógicos y curriculares exigidos desde el Ministerio de Educación.

En un comienzo los proyectos pedagógicos consideraron fuertemente las necesidades de aprendizajes de los alumnos y las posibilidades que el establecimiento puede ofrecer en función de la dotación docente disponible. En forma gradual los proyectos fueron evolucionando y consideraron con más importancia las demandas del nuevo currículo. En la evaluación del año 2001 esta alternativa fue mencionada como prioritaria solo por el 6,6\% de los directores. En el año 2005 el 79,7\% de los directivos señalaron que fue el principal criterio que tomaron en cuenta para la definición de las actividades de la JEC. Hoy día en la mayor parte de los establecimientos las actividades de los proyectos institucionales están relacionados con planes de mejoramiento de los aprendizajes y ejecución de distintos programas o proyectos en función de dicha meta.

Por último, en cuanto a los insumos, cabe señalar la relevancia dentro del diseño de la política que se le ha asignado al tiempo de trabajo colectivo docente. La JEC definió un total de 2 horas semanales para que los profesores desarrollen trabajo técnico-pedagógico colectivo, orientado a favorecer el mejoramiento del aprendizaje de los alumnos.

La puesta en marcha de la JEC no ha sido fácil para los establecimientos. Una vez superado los problemas de infraestructura la tarea principal es el diseño e implementación de su proyecto pedagógico donde los directivos deben lograr equilibrar la dotación docente, recursos disponibles y los nuevos requerimientos que plantea la JEC en cuanto implementación curricular y cumplimiento de horas.

\section{USO EFECTIVO DEL TIEMPO DE LA JEC}

A nivel general el uso del tiempo en los establecimientos educacionales se clasifica en tres grandes categorías: (a) total de la jornada, (b) tiempo no pedagógico (recreos y almuerzo) y (c) tiempo pedagógico, el que se divide en dos subcategorías: 
(i) plan de estudio, referido a los tiempos destinados a los sectores y subsectores curriculares y (ii) tiempo de libre disposición definido como aquel tiempo que por normativa el establecimiento puede definir libremente en función de su proyecto educativo y de los intereses de los estudiantes.

El Cuadro 1 da cuenta de las tendencias observables de distribución del tiempo en una muestra significativa de establecimientos basada en la última evaluación realizada con una muestra significativa de establecimientos (DESUC, 2005).

\section{Cuadro 1 -Tiempo general JEC normas de referencia en una semana enseñanza básica}

\begin{tabular}{|l|l|r|r|r|r|}
\hline \multicolumn{1}{|c|}{ Tiempo escolar } & \multicolumn{1}{|c|}{ Norma } & \multicolumn{1}{c|}{$\begin{array}{c}\text { Crítico } \\
(\%)\end{array}$} & $\begin{array}{c}\text { Bajo la } \\
\text { norma (\%) }\end{array}$ & $\begin{array}{c}\text { En la } \\
\text { norma }\end{array}$ & $\begin{array}{c}\text { Sobre la } \\
\text { norma (\%) }\end{array}$ \\
\hline Total jornada & 35 horas 25minutos & 7,5 & 21,4 & 45,7 & 25,3 \\
\hline Almuerzo & 3 horas 45minutos & 12,4 & 14,0 & 34,4 & 38,5 \\
\hline Recreo & 3 horas 10minutos & 23,0 & 45,2 & 10,3 & 21,4 \\
\hline Tiempo pedagógico & 28 horas 30minutos & 7,5 & 17,8 & 47,8 & 26,9 \\
\hline
\end{tabular}

Fuente: DESUC (2005).

Elaboración propia.

La gran mayoría de los establecimientos (82\% educación básica y 76\% enseñanza media) se ajustan o superan las horas previstas en la normativa, referidas al tiempo total de duración de la jornada escolar (en el rango y sobre el rango). En educación básica, más del $70 \%$ de los establecimientos cumple con la norma o está sobre ella en cuanto al tiempo pedagógico destinado a la implementación curricular.

Contrasta con esta situación el hecho de que el promedio destinado a almuerzo se ubica por sobre lo esperado normativamente, y a la inversa, el tiempo promedio destinado a recreos, se ubica bajo la referencia normativa. E1 38,5\% de los establecimientos de enseñanza básica y el 47,6\% de los de enseñanza media se ubican sobre la norma en relación al almuerzo. El 69\% de los establecimientos de enseñanza básica y el 63,2\% de los establecimientos de enseñanza media se ubican bajo la norma semanal de tiempo de recreo.

Estar bajo o sobre la norma en la distribución del tiempo es problemático ya que altera los ritmos y equilibrios planificados. En este sentido merecen una discusión los ahorros de tiempo asignado a los recreos ya que el juego y el descanso son tiempos necesarios para la formación y para garantizar atención. Del mismo modo, estar sobre la norma en los tiempos asignados a las disciplinas puede producir un efecto contraproducente por cansancio tanto a alumnos como profesores.

\section{USO DEL TIEMPO DE LIBRE DISPOSICIÓN}

Los establecimientos disponen de 8 horas semanales de libre disposición y que pueden organizar de acuerdo a las prioridades definidas por su proyecto pedagógico y los intereses de los estudiantes. 
En las evaluaciones se ha constatado que la mayor parte de los establecimientos se mantiene en el rango de la norma o cercano a la norma (6-8 horas semanales). Sólo un tercio $(35,1 \%)$ de los establecimientos destinan menos de 6 horas semanales a este tipo de actividades.

En cuanto al contenido o uso del tiempo adicional de libre disposición los establecimientos optan, principalmente, por desarrollar actividades relacionadas con los sectores de aprendizaje. La distribución obtenida se aprecia en el Cuadro 2.

Cuadro 2 - Uso del tiempo de libre disposición

\begin{tabular}{|c|c|c|c|}
\hline $\begin{array}{c}\text { Tipo de actividad } \\
\text { complementaria a principales } \\
\text { sectores y subsectores }\end{array}$ & $\begin{array}{l}\text { Establecimientos } \\
\text { que realizan la } \\
\text { actividad (\%) }\end{array}$ & $\begin{array}{l}\text { Promedio de horas } \\
\text { pedagógicas } \\
\text { realizadas en la } \\
\text { semana }\end{array}$ & $\begin{array}{c}\text { En el tiempo } \\
\text { total de libre } \\
\text { disposición (\%) }\end{array}$ \\
\hline $\begin{array}{l}\text { Taller lenguaje y } \\
\text { matemáticas (LEM) }\end{array}$ & 27,6 & 1,7 & 12,7 \\
\hline Reforzamiento (en general) & 25,6 & 1,5 & 10,4 \\
\hline Lenguaje y comunicación & 76,7 & 1,6 & 33,3 \\
\hline Matemáticas & 56,6 & 1,7 & 26,1 \\
\hline $\begin{array}{l}\text { Estudio y comprensión } \\
\text { de la naturaleza }\end{array}$ & 20,4 & 1,8 & 10,0 \\
\hline $\begin{array}{l}\text { Estudio y comprensión } \\
\text { de la sociedad }\end{array}$ & 5,9 & 1,6 & 2,6 \\
\hline Idioma extranjero & 10,3 & 1,4 & 3,9 \\
\hline Religión & 2,3 & 1,4 & 0,9 \\
\hline Total & --- & 1,6 & 100,0 \\
\hline
\end{tabular}

Fuente: DESUC (2005).

Elaboración propia.

Como se observa, las actividades que concentran la mayor cantidad de tiempo son las relacionadas con el sector de lenguaje y comunicación, realizadas por el $76,7 \%$ de los establecimientos, matemáticas (56,6\% de los establecimientos), reforzamiento en general ( $27,6 \%$ de los establecimientos) y actividades relacionadas con estudio y comprensión de la naturaleza realizadas por el $20,4 \%$ de los establecimientos de este nivel.

En síntesis, los establecimientos han organizado su tiempo siguiendo la normativa horaria planteada por la ley particularmente en relación con los tiempos pedagógicos y de implementación curricular. El cumplimiento de la norma no se observa en el caso del tiempo asignado a recreo, el que con mayor frecuencia resultó ser menor a lo definido. La situación da cuenta de una desvalorización de este tiempo no pedagógico y su aporte a los procesos educativos y de aprendizaje de los alumnos.

Por otra parte, se constata que el tiempo adicional se destina principalmente a actividades de reforzamiento en los sectores curriculares que son evaluados 
nacionalmente a través del Sistema de Medición que aplica anualmente el Ministerio de Educación (SIMCE/MINEDUC). Desde el punto de vista de los establecimientos es importante reforzar estas áreas de contenidos, las que son atendidas por los docentes disponibles en la escuela. Por ello son los mismos profesores quienes terminan realizando más horas de clases de matemáticas o de lenguaje afectando las posibilidades de innovación o la realización de actividades artísticas y deportivas complementarias.

La disminución de los tiempos de recreos y el reforzamiento de las materias con los mismos profesores en las horas adicionales ha llevado a un cansancio de profesores y alumnos. Este problema ha sido objeto de discusión pública y uno de los problemas planteados por los movimientos estudiantiles en sus movimientos de protestas donde destaca la realizada en el año 2006.

\section{EL TIEMPO EN LA SALA DE CLASES}

No existen estudios con muestras significativas de establecimientos y que analicen el impacto de la JEC en las prácticas pedagógicas y uso del tiempo en la sala de clases.

Una aproximación a la problemática la entregan los resultados de estudios que utilizan videos de clases filmados en el marco de la evaluación docente de los profesores que se desempeñan en el sistema público.

$\mathrm{El}$ año 2006 iniciamos un estudio que abordó las interacciones en la sala de clases y el uso o gestión del tiempo que realizan profesores y alumnos de segundo ciclo de enseñanza básica de establecimientos adscritos a la JEC. Este analizó 40 videos en los que se registran las clases presentadas por profesores de educación básica de los sectores de lenguaje y matemáticas. ${ }^{2}$ Se definieron y validaron categorías, las que fueron aplicadas a su vez en un segundo estudio que consideró otras 29 clases grabadas por profesores evaluados en el año 2008. ${ }^{3}$ Contamos así con una muestra de 69 casos y que da cuenta del mismo número de horas lectivas filmadas. Se siguió un procedimiento de observación continuo, aplicando una matriz de categorías cada 4 segundos durante los 40 minutos promedio de duración de cada una de ellas.

En el conjunto de las clases observadas el profesor ocupa la mayor parte del tiempo (52\%) y, por lo general, dirige su habla a la clase como un todo (46\% del tiempo equivalente a 17 minutos de la clase). El estilo de enseñanza que predomina no produce grandes diferencias entre los alumnos constituyendo al grupo curso en un "alumno promedio". Este es un estilo de enseñanza en la cual predomina una pedagogía directiva con poco diálogo y conexión con los estudiantes tal como ha sido demostrado en otros estudios (Martinic; Vergara, 2007).

2 Sergio Martinic (2006-2008), Representaciones sociales y gestión del tiempo en la sala de clases de establecimientos con Jornada Escolar Completa (FONDECYT n. 1060715).

3 Sergio Martinic (2008-2011), Interacciones pedagógicas y uso del tiempo en la sala de clases (PUC-CEPPE). 
En este tiempo, el 25\% del total de la clase el profesor da "instrucciones o información del contenido", lo que corresponde aproximadamente a un total de 10 minutos.

$\mathrm{Al}$ analizar el tipo de intervención de los profesores de lenguaje y matemáticas, se observa que la mayor parte se concentra en instrucciones o información relacionada con procedimientos a seguir y en la exposición de contenidos de la disciplina.

Ambas categorías se han definido como "tiempo instruccional" y corresponden a cerca del 39\% del tiempo de la clase. Se observa que predomina en el habla del profesor intervenciones que entregan instrucciones para organizar el trabajo o realizar la tarea en el marco de los contenidos de la clase. Estas intervenciones se realizan mayoritariamente en la fase de desarrollo de la clase. En el Cuadro 3 se describen las intervenciones del profesor y la proporción de tiempo que ocupan en la clase.

Cuadro 3 - Tipos de tiempo e intervenciones en discurso del profesor

\begin{tabular}{|l|r|r|r|r|}
\hline & Mínimo & Máximo & Media & Desviación típica \\
\hline Instruccional & $\mathbf{1 7 , 7 6}$ & $\mathbf{6 2 , 4 6}$ & $\mathbf{3 8 , 8 1}$ & $\mathbf{1 1 , 1 5}$ \\
\hline Expone & 0,20 & 39,40 & 13,74 & 9,76 \\
\hline Instrucción o información del contenido & 9,90 & 53,10 & 25,06 & 9,68 \\
\hline Interaccional & $\mathbf{6 , 2 3}$ & $\mathbf{5 3 , 3 1}$ & $\mathbf{2 4 , 8 5}$ & $\mathbf{9 , 8 0}$ \\
\hline Pregunta & 3,10 & 43,60 & 17,15 & 7,72 \\
\hline Responde contenido & 0,00 & 21,10 & 3,63 & 4,21 \\
\hline Evalúa + & 0,30 & 9,20 & 3,72 & 2,33 \\
\hline Evalúa - & 0,00 & 2,80 & 0,35 & 0,48 \\
\hline Regulativo & $\mathbf{2 , 5 0}$ & $\mathbf{6 2 , 7 3}$ & $\mathbf{3 1 , 0 7}$ & $\mathbf{1 4 , 0 7}$ \\
\hline Disciplina & 0,00 & 9,20 & 2,01 & 2,46 \\
\hline No interviene/observa & 2,50 & 61,20 & 29,06 & 14,67 \\
\hline Otra & $\mathbf{0 , 0 0}$ & $\mathbf{1 7 , 9 3}$ & $\mathbf{4 , 5 4}$ & $\mathbf{3 , 7 5}$ \\
\hline
\end{tabular}

Fuente: Elaboración propia a partir de datos recogidos en proyecto FONDECYT n. 1110601.

Elaboración propia.

Se observa que un $31 \%$ del tiempo del profesor se compone de intervenciones regulativas y que están dirigidas a disciplinar directamente a los alumnos o a observar las actividades sin una intervención verbal.

En cuanto a las intervenciones de los alumnos se observa que la mayor parte del tiempo se dedica a realizar tareas o a trabajar en función de actividades instruidas por los profesores. E1 18\% del tiempo de las intervenciones de los alumnos en lenguaje aluden a respuestas a preguntas del profesor. En matemáticas este alcanza el 13\% del tiempo de sus intervenciones. En ambos casos predominan respuestas con baja elaboración donde los alumnos se limitan a repetir o aprobar afirmaciones y contenidos enunciados por el profesor (idem). 
La participación y el protagonismo que tienen alumnos y profesores cambia a medida que transcurre la clase. En el Gráfico 1 se puede observar el tiempo que ocupan las intervenciones de profesores y alumnos durante la clase.

\section{Gráfico 1 - Hablante principal profesores matemáticas y lenguaje}

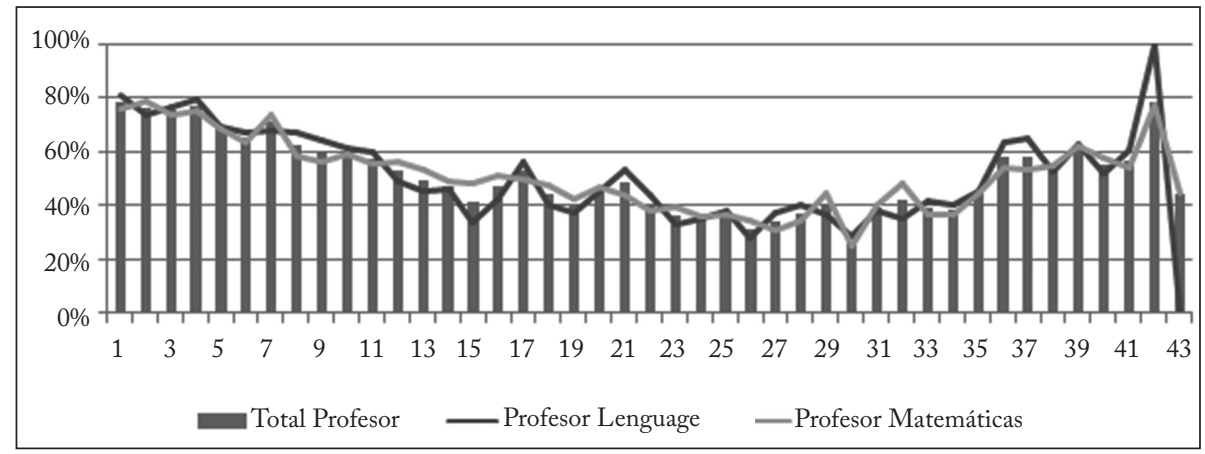

Fuente: Martinic; Huepe (2013, p. 130).

Elaboración propia.

Gráfico 2-Hablante principal alumnos en clases de lenguaje y matemáticas

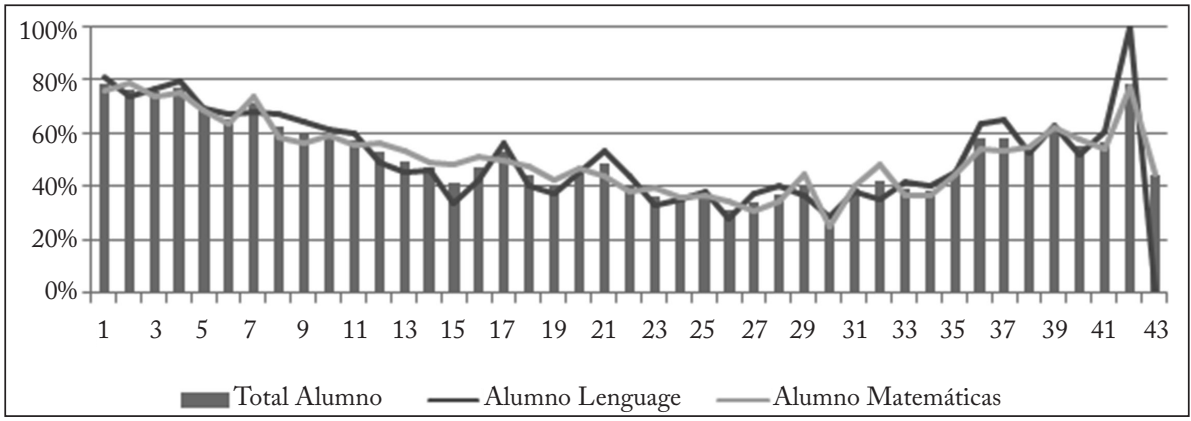

Fuente: Martinic; Huepe (2013 p. 130).

Elaboración propia.

Como se aprecia en los gráficos, los profesores ocupan la mayor parte del tiempo de habla durante los primeros siete minutos, para luego bajar a un tercio del tiempo entre los minutos 12 y 18 y en los últimos cinco minutos vuelve a tener un aumento en la fase de preparación del cierre.

En conclusión, predominan pautas de interacción altamente estructuradas por el profesor y donde los alumnos tienen un rol pasivo con intervenciones que ocupan poco tiempo en el conjunto de la clase. El estilo puede sintetizarse en que los profesores exponen, los estudiantes aplican y los profesores controlan dicha aplicación en el transcurso de la clase. Los usos del tiempo dan cuenta del protagonismo del profesor y de poco tiempo asignado a la voz y trabajos de los estudiantes.

Diversos estudios demuestran que estas interacciones son frecuentes en las escuelas que trabajan con niños provenientes de los sectores más pobres de la sociedad 
(Cruz et al. 2001; Martinic; Vergara, 2007; Draelents, 2009). Por lo general, los docentes de escuelas periféricas acentúan los aspectos disciplinarios por sobre los cognitivos, y en el aula ponen énfasis en el control del comportamiento de sus alumnos.

Se constata que no existen grandes cambios en la forma de organizar y gestionar el tiempo en la sala de clases de las escuelas observadas.

\section{EFECTOS DE LA JORNADA ESCOLAR EN LOS APRENDIZAJES}

Distintos estudios realizados por el MINEDUC y por otros investigadores han demostrado que la JEC tiene un impacto positivo en el logro académico de los estudiantes, medido través de la prueba SIMCE (Belleï, 2009; García, 2006; MINEDUC 2002; Valenzuela, 2005). Estos estudios son experimentos naturales, por lo que han debido controlar diversas variables que inducen sesgo, como variables del estudiante (género, porcentaje de repitencia, escolaridad de padre y madre, ingreso familiar, entre otras), variables del establecimiento (fuente de financiamiento, tamaño, cantidad de años implementando JEC, etc.) y otras variables como la elección de colegio por parte de las familias.

Hay diferencias en los coeficientes y en las magnitudes de los efectos, pero todos coinciden en que la JEC provoca un impacto positivo en el logro académico, que se traduce en un incremento de los puntajes SIMCE que oscila entre 2 a 8 puntos en lenguaje y 0 a 6 puntos en matemáticas.

Un estudio realizado por el MINEDUC (2002) indica que la extensión de la jornada en Chile ha significado para los establecimientos un incremento en los aprendizajes por año de 1.6 puntos en el SIMCE de lenguaje y 1.8 puntos en el SIMCE de matemáticas. Por su parte, Valenzuela (2005) y Belleï (2009) constatan que los establecimientos con más de 5 años en JEC tienen una mejoría de 5 puntos en promedio.

La magnitud de efecto de la JEC en el logro académico varía según la dependencia del establecimiento y el tipo de análisis. En educación básica (SIMCE cuarto y octavo básico), los establecimientos particulares subvencionados son los que más se benefician de la JEC (García, 2006; Valenzuela, 2005), mientras que en educación media (SIMCE segundo medio) se observa un mayor impacto de la JEC en los establecimientos rurales y municipales (Bellei, 2009). Por su parte, los análisis que controlan la no aleatoriedad de la asignación de establecimientos mediante el uso de técnicas de matching, tienden a arrojar efectos levemente mayores a los análisis de diferencias en diferencias simples, pero las diferencias no son sustanciales.

Estos resultados sobre el impacto de la JEC resultan ser consistentes con la percepción que tienen los actores de los establecimientos educacionales. Estudios realizados confirman una percepción positiva de los docentes sobre el desarrollo de capacidades de los alumnos. Las áreas en que identifican con mayores logros son las áreas artística, deportiva, manejo de tecnología y computación, formación valórica y capacidad de superación personal (DESUC, 2005).

Sin embargo, las áreas de desarrollo que se perciben más débiles son las asociadas a conocimientos y habilidades en matemáticas, lenguaje y el dominio del 
inglés. Es decir, los actores perciben efectos positivos en los alumnos pero estos no se relacionan necesariamente con los aprendizajes de las materias básicas. Los profesores atribuyen los problemas de aprendizaje al bajo capital cultural de las familias, lo que no logra ser contrarrestado por la política de la JEC (Martinic; Huepe; Madrid, 2008).

Estos resultados sugieren que la relación entre tiempo y aprendizaje requiere de una serie de consideraciones. En primer lugar, existe una amplia discusión sobre el concepto objetivo y absoluto de tiempo y que atribuye a la suma o resta de horas de clases un incremento proporcional y directo en los aprendizajes (Husti, 1992; Metzker, 2003; Sue, 2005). Mientras más amplias son las categorías de análisis (por ejemplo, días de clases; horas anuales de instrucción) mayor será la relación entre la asignación de tiempo y los resultados de aprendizaje. Sin embargo, al focalizar el análisis o aplicar categorías más micro la relación se hace más compleja.

Por otra parte, los impactos de la política son mayores cuando se actúa sobre un conjunto de factores y no solo sobre la asignación de tiempo. La mayor parte de los estudios concluyen que si no se actúa sobre el conjunto del sistema de variables que inciden en el aprendizaje, el efecto de los cambios en la jornada escolar es prácticamente nulo (Cotton; Savard, 1990; Martinic; Huepe; Madrid, 2008; Metzker, 2003; Rodriguez, 2009; Tenti, 2010).

Por último, se ha demostrado que el mayor tiempo escolar genera mejores aprendizajes siempre y cuando sea acompañado de tiempo efectivo dedicado a la tarea por parte de los estudiantes y por un reordenamiento de los medios didácticos, de las estructuras curriculares y de los contenidos educativos que se enseñan (Karampelas, 2005; Metzker, 2003).

En el país se ha realizado una amplia discusión sobre el impacto que tiene la Jornada Escolar Extendida en la calidad de los procesos educativos y de los aprendizajes. Esta discusión ha destacado los problemas pedagógicos que tienen la implementación de la JEC y las dificultades que existen en la cultura escolar para organizar el tiempo y la pedagogía de modo más flexible e innovador.

Se ha cumplido una fase de instalación y de preparación de los establecimientos y se debe pasar a otra de gestión relacionada con la calidad pedagógica de la gestión y uso del tiempo en los establecimientos.

\section{CONCLUSIONES Y DISCUSIÓN FINAL}

La falta de tiempo en las escuelas y las pocas horas dedicadas al estudio afectan, de un modo particular, a los niños que no pueden compensar dichas carencias con espacio, tiempo y estímulo para el estudio en sus realidades familiares.

A modo de hipótesis, sostenemos que el cambio en la jornada escolar debe ir acompañado de un cambio en el modo de conceptualizar, gestionar y utilizar el tiempo en el establecimiento y en la sala de clases.

Se ha demostrado que un aumento cuantitativo en el número de horas no basta para mejorar la calidad de los resultados. En efecto, cualquier innovación en los tiempos escolares debe ir acompañada de cambios en las prácticas pedagógicas y en los recursos disponibles para el profesor. 
El tiempo escolar suele organizarse en función de diferentes actividades. La mayor parte del tiempo está orientada al plan común curricular, tiempo adicional y complementario orientado al desarrollo de actividades que responden a los intereses de los propios estudiantes y tiempo para el trabajo docente en equipo o individual. La cantidad de estos tiempos como la relación que existe entre ellos es objeto de discusión en América Latina y da origen a diversas modalidades de organización. Del mismo modo, se trata de tiempos y espacios diferentes que requieren, a su vez, metodologías y actores distintos. La norma horaria debe compatibilizar la enseñanza del curriculum con el desarrollo de actividades de libre disposición y que desarrollan habilidades sociales y culturales entre los estudiantes.

La flexibilidad del tiempo educacional es una característica importante para las escuelas que atienden a estudiantes de secundaria o en riesgo de fracaso escolar. Las escuelas que enseñan cuando los estudiantes están disponibles para ser enseñados y que reconocen variaciones en el ritmo de aprendizaje de los estudiantes son importantes complementos al sistema educativo.

Las decisiones en relación a la cantidad y calidad del tiempo debe ser producto de un debate profundo que involucre a toda la comunidad educativa en torno a una interpretación amplia del problema. Después de todo, cualquier cambio en los horarios afecta a la familia y a toda la comunidad.

La ampliación del tiempo escolar tiene otros beneficios sociales y que trasciende al campo escolar. Las familias modernas son más pequeñas y la mujer se incorpora crecientemente al mundo del trabajo. La jornada continua facilita la compatibilidad de la vida familiar y la vida escolar.

La ampliación de jornada permite aumentar la participación de los padres en el centro educativo, porque este se transforma en institución que ofrece, además de la educación como tal, actividades culturales que integran a la comunidad. Así, en Europa, la escuela se ha transformado en un factor de integración social, cultural y comunitaria.

Los estudios recomiendan que la flexibilidad también se exprese en un cambio del polo del control de gestión incorporando a los actores del sistema educativo. La participación de los alumnos en las decisiones y en la posibilidad de elección de lo que se quiere hacer es una medida muy importante en términos de las repercusiones ligadas a la convivencia institucional.

Por otra parte, la extensión de jornada y existencia de tiempo adicional ofrece una oportunidad para generar nuevas relaciones entre la escuela y la comunidad y la participación de los padres. Por ejemplo, en la provincia de Buenos Aires el tiempo adicional se gestiona a través de una comisión integrada por docentes, personal directivo, alumnos, personal auxiliar y padres. Ellos son los que controlan el desarrollo de la oferta de doble jornada realizando un seguimiento que incluye reuniones periódicas y la elaboración de informes acerca de la marcha institucional de estas escuelas.

La extensión de jornada permite el aprendizaje de estrategias de ocio activo en los alumnos. La utilización del tiempo libre de forma activa y positiva se ha convertido en una necesidad básica de la persona humana. El desarrollo tecnológico cada vez nos proporciona mayor tiempo de ocio y los alumnos deben aprender 
progresivamente a organizar ese tiempo de modo inteligente, al margen de la estructura de horarios y contenidos de la jornada lectiva.

La participación en actividades no académicas (extraescolares) ayuda a conseguir este objetivo, al poseer un grado de estructuración menor y una mayor adaptación a los intereses personales del alumno. En esta dinámica, el estudiante decide qué le interesa, en qué y cuándo participa.

Estos mismos estudios enfatizan que la extensión de jornada revalida al individuo a través de su participación en actividades artísticas que ayudan, principalmente, a los alumnos que cuentan con problemas de participación, sobre todo en una escuela que atiende a una comunidad socialmente deprimida.

Finalmente es importante destacar la importancia de la extensión del tiempo para el trabajo docente y su desarrollo profesional. Es una aspiración compartida por los docentes en América Latina el contar con más tiempo no lectivo. Este tiempo puede ser dedicado a trabajo personal y colectivo en función de los aprendizajes de los estudiantes y resolución de problemas del quehacer pedagógico. La extensión de jornada ofrece una oportunidad para el desarrollo profesional.

\section{REFERENCIAS}

Abadzi, H. Absenteeism and beyond: instructional time loss and consequences. Washington, DC: Global Evaluation Division (IEGSG), Independent Evaluation Group (IEG). Policy Research Working Paper n. 4.376, p. 96, 2007.

Instructional time loss in developing countries: concepts, measurement, and implications. The World Bank Research Observer, Washington, DC: The World Bank; Washington: Oxford University Press and the International Bank for Reconstruction and Development/The World Bank, v. 24, n. 2, p. 267-290, 2009.

Aronson, J.; Zimmerman, J.; Carlos, L. Improving student achievement by extending school: is it just a matter of time? American Education, San Francisco, p. 1-9, 1998. Disponible en: <http://www.wested.org/online_pubs/po-98-02.pdf>. Acceso en: 20 oct. 2012.

BELLEÏ, C. Does lengthening the school day increase students' academic achievement? Results from a natural experiment in Chile. Economics of Education Review, New York: Elsevier Educational Research Pro, v. 28, n. 5, p. 629-640, 2009.

B Loom, B. An introduction to mastery learning. In: B and mastery learning. New York: Holt, Rinehart and Winston, 1974.

Brunner, J. J.; Elacqua, G. Calidad de la educación. Claves para el debate. Santiago: Ril Editores, 2006.

Cerdan-Infantes, P.; Vermeersch, C. More time is better: an evaluation of the full time school program in Uruguay. Washington, DC: World Bank Policy Research Working Paper 416, 2007. Disponible en: <http://papers.ssrn.com/sol3/papers. cfm?abstract_id=970809>. Acceso en: 12 nov. 2012.

Cotton, K.; Savard, W. G. Time factors in learning. Research on School Effectiveness Project: topic summary report. Close up n. 8, Portland, Oregon: Northwest Regional Educational Lab., 1990. 
Cox, C. (Ed.). Politicas educacionales en el cambio de siglo. La reforma del sitema escolar chileno. Santiago: Editorial Universitaria, 2005.

Cruz, M.; Scheuer, N.; Caino, G.; Huarte, M.; Baudino, V.; Ayastuy, R. El discurso en clase de maestros de nivel primario en distintos sectores socioculturales. Estudios Pedagógicos, Valdivia, Chile: Universidad Austral de Chile, n. 27, p. 2-41, 2001. Disponible en: <http://www.scielo.cl/scielo.php?script=sci_arttext\&pid=S071807052001000100002\&lng=es\&nrm=iso >. Acceso en: 14 enero 2013.

Delhaxhe, A. Le temps comme unité d'analyse dans la recherche sur l'enseignement. Revue Francaise de Pédagogie, Paris: Institut National de Recherche Pédagogique (INRP), n. 118, p. 107-25, janv./mars. 1997.

Desuc - Dirección de Estudios Sociológicos. Informe final estudio Evaluación Jornada Escolar Completa. Santiago: Pontificia Universidad Católica de Chile; Ministerio de Educación de Chile, 2005.

Draelents, H. Reforme pédagogique et légitimation. Bruxelles: De Boeck, 2009.

Eren, O.; Millimet, D. L. Time to learn? The organizational structure of schools and student achievement. Empirical Economics, v. 32, n. 2-3, p. 301-332, 2007.

Friese, H. Le temps-discours, les temps-images. Pluralisation et ouverture de l'organisation temporelle de la vie quotidienne. Politix, Paris: Armand Colin, v. 10, n. 39, p. 39-64, 1997.

García, A. Evaluación del impacto de la Jornada Escolar Completa. Santiago: Universidad de Chile; Facultad de Economia, 2006. Documento de trabajo.

Husti, A. Del tiempo escolar uniforme a la planificación móvil del tiempo. Revista de Educación, Madrid: Ministerio de Educación y Cultura, n. 298, p. 271-305, 1992.

Karampelas, K. Redesigning time management in response to educational change: teachers perception towards timing, instruction and further education. In: CONFERENCE OF the International Congress for School Improvement. Barcelona, 2-5 enero 2005. Martinic, S.; Huepe, D. Uso del tiempo e interacciones en la sala de clases. Un estudio de casos en Chile. Revista Pro-Posiçôes, Campinas: UNICAMP, v. 24, n.1 (70), 2013. Disponible en: <http://www.scielo.br/scielo.php?script=sci_arttext\&pid=S0103$73072013000100009 \& \operatorname{lng}=$ pt\&nrm=iso\&tlng=es >. Acesso en: 20 dic. 2014.

.; Huepe, D.; Madrid, A.Jornada Escolar Completa en Chile. Representaciones sociales sobre sus efectos en los aprendizajes. Revista Iberoamericana de Evaluación Educativa, Madrid: Red Iberoamericana de Investigación sobre Cambio y Eficacia Escolar, v. 1, n. 1, p. 124-139, 2008.

.; Vergara, C. Gestión del tiempo e interacción del profesor-alumno en la sala de clases de establecimientos de Jornada Escolar Completa en Chile. Revista Electrónica Iberoamericana sobre Calidad, Eficacia y Cambio en Educación, Madrid: Red Iberoamericana de Investigación sobre Cambio y Eficacia Escolar, v. 5, n. 5, p. 3-20, dic. 2007. Disponible en: <http://www.rinace.net/arts/vol5num5e/art1.pdf. 2007>. Acceso en: 18 oct. 2012.

Metzker, B. Time and learning. ERIC digest. Digest number 166, [s.1.]: ERIC Clearinghouse on Educational Management Eugene OR 2003. Disponible en: <http:// files.eric.ed.gov/fulltext/ED474260.pdf >. Acceso en: 15 oct. 2012.

Mineduc. Prueba SIMCE $2^{\circ}$ medio 2001. Análisis de resultados. Santiago: Ministerio de Educacion de Chile; División de Planificación y Presupuesto, 2002. 
Мокате, K. El monitoreo y la evaluación: herramientas indispensables de la gerencia social. Washington: INDES, ago. 2000. Documento de trabajo.

Or Kin, K. Does lengthening the school day improve students' academic achievement? Evidence from a natural experiment in Ethiopia. Londres: Young Lives project local 2011. Disponible en: <https://editorialexpress.com/cgi- bin/conference/download. cgi?db_name=CSAE2012\&paper_id=648>. Acceso en: 12 nov. 2012.

Redd, Z.; Boccanfuso, C.; Walker, K.; Princiotta, D.; Knewstub, D.; Moore, K. Expanding time for learning both inside and outside the classroom: a review of the evidence base by. Editorial Child Trends, ago. 2012. Disponible en: <http://www. wallacefoundation.org/knowledge-center/summer-and-extended-learning-time/ extended-learning-time/Documents/Expanding-Time for-Learning-Both-Inside-andOutside-the-Classroom.pdf>. Acceso en: 20 nov. 2012.

Rodriguez, C. Comment. "Do longer school days have enduring educational, occupational, or income effects? A natural experiment in Buenos Aires, Argentina”. Economia, Buenos Aires: Brookings Institution Press, v. 10, n. 1, p. 34-38, 2009.

Simce. Resultados Nacionales 2008. Santiago: MINEDUC, Unidad de Currículum y Evaluación (UCE), mayo 2009.

SuE, R. Les temps nouveaux de l'éducation. In: .; Caccia, M.-F. (Eds.). Autres temps, autre école. Impact et enjeux des rythmes scolaires. Paris: Retz, 2005. Disponible en: <http://www.scienceshumaines.com/-0aautres-temps-2c-autre-ecole-impact-etenjeux-des-rythmes-scolaires-0a_fr_5235.html>. Acceso en: 3 dic. 2012.

Tenti, E. Estado del arte: escolaridad primaria y jornada escolar en el contexto internacional. Estudio de casos en Europa y América Latina. Buenos Aires: IIEPE; UNESCO, 2010.

Valenzuela, J. P. Partial evaluation of a big reform in the Chilean education system: from a half day to a full day schooling. 2005. Tesis (Ph.D. en Economía) - University of Michigan, 2005.

Vercellino, S. La ampliación del tiempo escolar: ¿se modifican los componentes duros del formato escolar? Revisión bibliográfica sobre estas temáticas. Revista Electrónica Educare, San Jose, Costa Rica: Universidad Nacional de Costa Rica, v. 16, n. 3, p. 9-36, 2012. Disponible en: <http://www.revistas.una.ac.cr/index.php/EDUCARE/issue/ current>. Acceso en: 30 enero 2013.

Wubbels, T.; Brekelmans, M. Two decades of research on teacher-student relationships in class. International Journal of Educational Research, New York: Elsevier,n. 43,p. 6-25, 2005.

\section{SOBRE EL AUTOR}

Sergio Martinic es Ph.D. en sociología por la Université Catholique de Louvain (Bélgica). Profesor asociado de la Pontificia Universidad Católica de Chile (Chile).

E-mail:smartini@uc.cl 